\title{
Adaptive Morphology using Tensor-Based Elliptical Structuring Elements
}

\author{
Anders Landström*, Matthew J. Thurley \\ Department of Computer Science, Electrical and Space Engineering, \\ Luleå University of Technology, SE-971 87 Luleå, Sweden
}

\begin{abstract}
Mathematical Morphology is a common strategy for non-linear filtering of image data. In its traditional form the filters used, known as structuring elements, have constant shape once set. Such rigid structuring elements are excellent for detecting patterns of a specific shape, but risk destroying valuable information in the data as they do not adapt in any way to its structure.

We present a novel method for adaptive morphological filtering where the local structure tensor, a well-known method for estimation of structure within image data, is used to construct adaptive elliptical structuring elements which vary from pixel to pixel depending on the local image structure. More specifically, their shape varies from lines in regions of strong single-directional characteristics to disks at locations where the data has no prevalent direction.
\end{abstract}

Keywords: Mathematical morphology, Local structure tensor, Adaptive morphology, Spatially-variant morphology

\section{Introduction}

\subsection{Background}

Mathematical morphology, originally developed by Matheron (1975) and Serra (1982), is a powerful method for filtering highly non-linear image data. It is based on kernels called structuring elements, which are used to probe the image by considering pixel values within the resulting neighborhoods. More specifically, the two basic operations erosion and dilation extracts the minimum or maximum value, respectively, within the neighborhood defined by the structuring element.

Traditional mathematical morphology uses one single userdefined structuring element for the whole image. This strategy enables efficient implementation and is very useful for detecting objects of a certain size and shape, but selecting a suitable structuring element becomes more challenging when size and shape of objects in the image are more varying. The challenge posed by objects of different sizes can be handled by iteratively processing the image using a set of differently sized structuring elements, as is done e.g. when calculating morphological granulometries (Matheron, 1975) or the ultimate opening (Beucher, 2007), but this requires that the whole image is processed multiple times. Consequently, there is an ongoing interest in more adaptive approaches to mathematical morphology, largely based on theory originally introduced by Serra (1988).

As pointed out by Roerdink (2009), there is ambiguity in literature regarding the terms associated with adaptive or spatially-variant morphology. The terms are sometimes used interchangeably, but can also be used to separate between different approaches. Roerdink identifies two major categories of generalizations of classical mathematical morphology;

\footnotetext{
*Corresponding author. Tel. +4692049 3010, fax +46920492801

Email addresses: anders.p.landstrom@ltu.se (Anders Landström), matthew . thurley@ltu. se (Matthew J. Thurley)
}

1. Group morphology, where translation invariance is replaced by other forms of invariance, and

2. Adaptive morphology, where structuring elements depend on position or the input image.

Regarding group morphology, Roerdink (1988; 2000) provides a framework for extending morphological operations to other invariant mathematical groups such as motion-invariant grayscale operators and gray-scale operators on the sphere. Within adaptive morphology, Roerdink (2009) defines two separate forms of adaptivity: (a) letting the structuring element depend on the location in the image only (location-adaptive morphology), and (b) letting the structuring element depend on the actual image values (input-adaptive morphology). In this work we will concentrate on input-adaptive morphology, setting the shapes of the structuring elements from the image structure. Once set, however, structuring elements remain fixed for each pixel and can then be considered depending on the location in the image.

Input-adaptive morphology within the concept of binary images was extended by Charif-Chefchaouni and Schonfeld (1994) based on the work by Serra (1988). Bouaynaya and Schonfeld (2006) continued the development of these ideas by defining input-adaptive mathematical morphology for grayscale images using the Umbra transform, extending the formal theoretical framework even further. Roerdink (2009) later complemented the work by Bouaynaya and Schonfeld by showing that the same structuring element must be used for both erosion and dilation at an image pixel in order to achieve adjunction - a necessary relation between the two in order to achieve the defining properties of morphological operations (Serra, 2005). For more extensive background and theory on the topic of adaptive morphology, we refer the reader to Bouaynaya et al. (2008; 2008). For a review of current methods within the subject, the 
reader is referred to Maragos and Vachier (2009).

\subsection{Related Work}

Several approaches to input-adaptive mathematical morphology have been suggested. Shih and Cheng (2004) use adaptive elliptical structuring elements for edge linking in binary data, setting the parameters of the ellipses from local path curvature. Shih and Gaddipati (2003) presents a framework for general sweep mathematical morphology, where structuring elements vary along a given path (a closed curve or the boundary of a closed object).

Lerallut et al. (2007) introduce the concept of morphological amoebas, where a weighted distance measure is used to adapt the structuring element so that its growth across strong gradients is prevented. Recently, Curic et al. (2012) present salience adaptive structuring elements, which are less flexible than morphological amoebas and less affected by noise. Debayle and Pinoli (2005) define structuring elements based on a similarity measure, so that all pixels within a similar (as defined by the similarity measure) connected region share the same (noncentered) structuring element.

Verdú-Monedero et al. use average diffused squared gradient fields to obtain angles for line-shaped structuring elements (Verdú-Monedero and Angulo, 2008; Verdú-Monedero et al., 2009, 2011). An interpolation by diffusion sets angles for pixels further away from the actual directional variations (i.e. edges) within the image. Tankyevych et al. (2009) use principal value analysis of the Hessian matrix of 3D voxels to obtain directions for 3D line structuring elements. To detect objects of different radii, multiple scales are used. Breuß et al. (2007) use tensors to approximate angles for continuous morphology, achieved by solving partial differential equations for diffusion.

In addition to the above, there is work on efficient implementation of size-invariant morphological operations without concern to how the per-pixel sizes are retrieved. Cuisenaire (2006) presents an implementation for differently sized structuring elements shaped as balls of a selected metric, and Dokládal and Dokládalová (2008) shows how rectangular structuring elements of varying sizes can be applied.

For the case of adjunct morphological operations, Lerallut et al. (2007) and Tankyevych et al. (2009) have demonstrated how such operations can be efficiently implemented for adaptive structuring elements.

\subsection{Contribution}

In this work, we define a framework for input-adaptive morphology, based on the Local Structure Tensor (LST), where adaptive elliptical structuring elements range from lines to disks depending on the local image structure. Capturing the eigenvalues as well as the eigenvectors of the LST within the shape of the structuring element allows the method to adapt not only to the orientation within the data, but also to its dominancy (the degree of anisotropy). This is important because any deviation in the data (such as noise) will yield an orientation, even when there is no true structure in that region of the image.

Allowing the structuring element shape itself to vary depending on the eigenvalues of the LST avoids a distinct geometrical bias from the structuring element itself. Fixing the shape of the structuring element to lines, for instance, is highly likely to introduce artificial lines in the filtered image, since extreme values (e.g. noise) will cause distinct straight edges to appear in the result.

The orientation angles provided by the LST are equivalent to the angles given by gradient fields (Rieger and Van Vliet, 2004) and have thereby been implicitly used by Verdú-Monedero et al. for setting the orientations of line structuring elements (VerdúMonedero and Angulo, 2008; Verdú-Monedero et al., 2009, 2011). In their work, more certain orientation information is interpolated by diffusion into regions where the gradient values are low.

However, by explicitly using the LST we can take the relation between its eigenvalues into account instead of interpolating angles into non-varying image regions. This strategy does not impose an orientation where there is no prevalent direction in the data and therefore avoids introducing a geometrical bias. Interpolation is also generally quite time-consuming, while the LST contains orientation dominancy information in its eigenvalues - which in lower dimensions can be efficiently calculated from closed-form expressions. The LST has previously been used explicitly by Breuß et al. (2007) for (diffusion-based) mathematical morphology, but as well without regard to its eigenvalues.

\section{Method}

\subsection{The Local Structure Tensor}

Let $\mathbf{x}=\left(\begin{array}{ll}x_{1} & x_{2}\end{array}\right)^{T}$ denote pixel coordinates and $f(\mathbf{x})$ the corresponding gray-scale value. The Local Structure Tensor (LST) $\mathbf{T}(\mathbf{x})$, representing local directional features in the data, is then given by the $2 \times 2$ matrix

$$
\mathbf{T}(\mathbf{x})=\left(\begin{array}{ll}
T_{X_{1} X_{1}} & T_{X_{1} X_{2}} \\
T_{X_{1} X_{2}} & T_{X_{2} X_{2}}
\end{array}\right)(\mathbf{x})=G_{\sigma} *\left(\nabla f(\mathbf{x}) \nabla^{\mathrm{T}} f(\mathbf{x})\right),
$$

where $\nabla=\left(\frac{\partial}{\partial x_{1}} \frac{\partial}{\partial x_{2}}\right)^{\mathrm{T}}$ and $G_{\sigma}$ denotes a Gaussian filter kernel with standard deviation $\sigma$. The resulting smoothing regularizes the matrix (Cammoun et al., 2009). In practice, we estimate the image gradient by applying standard $3 \times 3$ pixel Scharr filters operating on a slightly smoothed version of the input image (produced by applying a small Gaussian filter with low standard deviation $\sigma^{\prime}$ to the input image $f$ ), while $\sigma$ for the tensor smoothing is defined by assigning a filter bandwidth radius $r_{w}$. More specifically, $\sigma$ is given by

$$
\sigma=\frac{r_{w}}{\sqrt{2 \ln 2}}
$$

i.e. $G_{\sigma}$ decreases to half of its maximum value at distance $r_{w}$ from its center.

For each pixel $\mathbf{x}$, we then consider the eigenvalues $\lambda_{1}(\mathbf{x})$ and $\lambda_{2}(\mathbf{x})\left(\lambda_{1}(\mathbf{x}) \geq \lambda_{2}(\mathbf{x})\right)$ and the corresponding eigenvectors $\mathbf{e}_{1}(\mathbf{x})$ and $\mathbf{e}_{2}(\mathbf{x})$ of the symmetric LST $\mathbf{T}(\mathbf{x})$. These provide information about edges present in the data. Eigenvalues can be interpreted as follows: 
$\lambda_{1} \approx \lambda_{2} \gg 0: \quad$ No dominant direction (edge crossing or point),

$\lambda_{1} \gg \lambda_{2} \approx 0$ : Strong dominant direction (edge),

$\lambda_{1} \approx \lambda_{2} \approx 0: \quad$ No dominant direction (no edge).

The eigenvector $\mathbf{e}_{1}(\mathbf{x})$ represents the local dominant direction of variation in the data. Hence $\mathbf{e}_{2}(\mathbf{x})$, being orthogonal to $\mathbf{e}_{1}(\mathbf{x})$, represents the direction of the smallest variation in the region (Cammoun et al., 2009).

\subsection{Adaptive Elliptical Structuring Elements}

We define the (solid) elliptical structuring element $E(a, b, \phi)$, where $a$ is the semi-major axis, $b$ the semi-minor axis, and $\phi$ the orientation (see Fig. 1). Each pixel $\mathbf{x}$ in the image $f$ is then assigned a structuring element. Hence, we have $a=a(\mathbf{x}), b=$ $b(\mathbf{x})$, and $\phi=\phi(\mathbf{x})$.

The axes $a(\mathbf{x})$ and $b(\mathbf{x})$ are set from the eigenvalues of $\mathbf{T}(\mathbf{x})$ by the expressions

$$
\begin{aligned}
& a(\mathbf{x})=\frac{\lambda_{1}(\mathbf{x})}{\lambda_{1}(\mathbf{x})+\lambda_{2}(\mathbf{x})} \cdot M, \\
& b(\mathbf{x})=\frac{\lambda_{2}(\mathbf{x})}{\lambda_{1}(\mathbf{x})+\lambda_{2}(\mathbf{x})} \cdot M,
\end{aligned}
$$

where $M$ denotes the maximum allowed semi-major axis. Divisions by zero and perfectly smooth regions are handled by adding a small positive number to the eigenvalues (small enough to be neglected for any other purpose, i.e. machine epsilon). For all values of $\lambda_{1}(\mathbf{x})$ and $\lambda_{2}(\mathbf{x})$ we have $a(\mathbf{x})+b(\mathbf{x})=M$ and $0 \leq b(\mathbf{x}) \leq a(\mathbf{x}) \leq M$. The structuring elements will dynamically range from lines of length $M$ where $\lambda_{1}(\mathbf{x}) \gg \lambda_{2}(\mathbf{x}) \approx$ 0 , i.e. near strong dominant edges in the data, to disks with radius $M / 2$ where $\lambda_{1}(\mathbf{x}) \approx \lambda_{2}(\mathbf{x})$, i.e. where no single direction represents the local image structure (see Section 2.1). The orientation $\phi(\mathbf{x})$ is retrieved from the corresponding eigenvectors by

$$
\phi(\mathbf{x})=\left\{\begin{array}{cc}
\arctan \left(\frac{e_{2, x_{2}}(\mathbf{x})}{e_{2, x_{1}}(\mathbf{x})}\right), & e_{2, x_{1}}(\mathbf{x}) \neq 0, \\
\frac{\pi}{2}, & e_{2, x_{1}}(\mathbf{x})=0
\end{array}\right.
$$

where $e_{2, x_{1}}(\mathbf{x})$ and $e_{2, x_{2}}(\mathbf{x})$ denote the components of the eigenvector $\mathbf{e}_{2}(\mathbf{x})$.

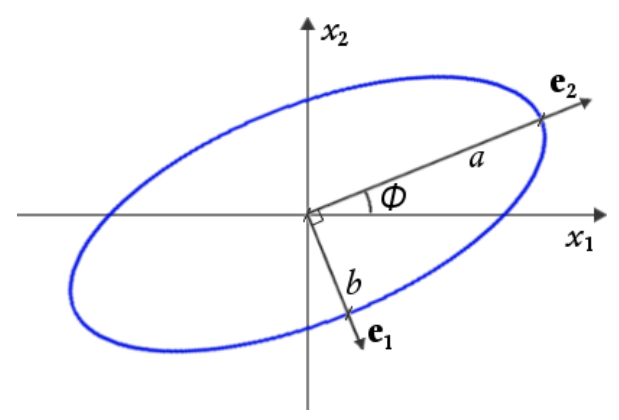

Figure 1: Ellipse parameters $a, b$, and $\phi$, and their relation to the eigenvectors $\mathbf{e}_{1}$ and $\mathbf{e}_{2}$ of the LST.
Given the image $f$ and the parameters $M$ and $r_{w}$, the values of $a(\mathbf{x}), b(\mathbf{x})$, and $\phi(\mathbf{x})$ for the pixel $\mathbf{x}$ are thereby uniquely defined. To further reduce the complexity we suggest setting $r_{w}=M$, ending up with only one parameter, but this can of course be adjusted as needed based on the size and nature of the features of interest. This will be further investigated in Section 4.1.

\subsection{Morphological Operations}

For simplicity, we here introduce the notation

$$
N_{E}(\mathbf{x})=E_{\mathbf{x}}(a(\mathbf{x}), b(\mathbf{x}), \phi(\mathbf{x}))
$$

for the elliptical neighborhood surrounding the pixel $\mathbf{x}$. The pixel-dependent ellipse parameters $a(\mathbf{x}), b(\mathbf{x})$, and $\phi(\mathbf{x})$ are calculated from the local image structure according to Eqs. (1)(5), and the $\mathbf{x}$ subscript denotes that the elliptical structuring element has been translated to the pixel $\mathbf{x}$. We then define the morphological operations erosion $\left(\varepsilon_{E}\right)$ and dilation $\left(\delta_{E}\right)$ operating on the image $f$ by

$$
\begin{aligned}
& \varepsilon_{E}(f)=\bigwedge_{\mathbf{y}: \mathbf{y} \in N_{E}(\mathbf{x})} f(\mathbf{y}) \quad \forall \mathbf{x} \in \mathcal{D}(f), \\
& \delta_{E}(f)=\bigvee_{\mathbf{y}: \mathbf{x} \in N_{E}(\mathbf{y})} f(\mathbf{y}) \quad \forall \mathbf{x} \in \mathcal{D}(f),
\end{aligned}
$$

where $\bigwedge$ and $\bigvee$ denotes the minimum and maximum operators, respectively, and $\mathcal{D}(f)$ is the support domain of the image $f$.

The operations $\varepsilon_{E}$ and $\delta_{E}$ are adjunct since the structuring elements are defined once from the same input image (referred to as the "pilot image" by Lerallut, 2007) and thereafter remain fixed throughout the subsequent morphological operations (Roerdink, 2009). That is,

$$
\delta(f) \leq g \Longleftrightarrow f \leq \varepsilon(g)
$$

for any functions $f$ and $g$. We can thereby define the operations

$$
\begin{aligned}
& \gamma_{E}(f)=\left(\delta_{E} \circ \varepsilon_{E}\right)(f), \\
& \psi_{E}(f)=\left(\varepsilon_{E} \circ \delta_{E}\right)(f)
\end{aligned}
$$

to be the morphological opening and closing, respectively, of the gray-scale image $f$.

\section{Implementation}

The presented method was tested by a fairly straight-forward implementation in OpenCV v.2.3 (2011). The program can be summarized as follows:

1. Pre-calculate relative pixel index lists for the expected elliptical structuring elements, i.e. the index shifts for the leftmost and rightmost border pixels (relative to the center pixel) for each row of the discretized structuring element. All of the required structuring elements, as defined by $M$, are then stored in a corresponding 3D Look-Up Table $L U T_{E}(a, b, \phi)$ based on semi-major axes $a \in[0, M]$, semi-minor axes $b \in[0, a]$, and orientations $\phi \in[0, \pi)$. In practice, due to the symmetry of the ellipses, only half of the relative indices need to be stored. The other half can then be easily obtained by inverting the coordinates. 
2. Estimate the image gradient $\nabla f(\mathbf{x})$ using $3 \times 3$ pixel Scharr filters on a pre-smoothed version of the input image, obtained using a small Gaussian filter with standard deviation $\sigma^{\prime}=0.95$. Then calculate the LST for each pixel according to Eq. (1), defining the standard deviation $\sigma$ by setting a filter bandwidth radius $r_{w}$.

3. Obtain axis lengths and orientation for each pixel from the eigenvalues and eigenvectors of the corresponding LST, using Eqs. (3)-(5).

4. Perform an erosion or a dilation, or a combination of the two (an opening or a closing), using the elliptical structuring elements defined for each pixel $\mathbf{x}$. More specifically, the elliptical neighborhoods provided by $\operatorname{LUT}_{E}(a(\mathbf{x}), b(\mathbf{x}), \phi(\mathbf{x}))$ are used to perform the operations given by Eqs. (7) and (8). In practice, the dilation is implemented as suggested by Lerallut (2007), i.e.

$$
\begin{aligned}
& {\left[\delta_{E}(f)\right](\mathbf{y})=\bigvee\left(\left[\delta_{E}(f)\right](\mathbf{y}),\right.}f(\mathbf{x})), \\
& \forall \mathbf{y} \in N_{E}(\mathbf{x}), \forall \mathbf{x} \in \mathcal{D}(f) .
\end{aligned}
$$

\section{Results}

\subsection{Choice of Parameters}

The impact of the two required input parameters $M$ (maximum semi-major axis) and $r_{w}$ (filter bandwidth for tensor estimation) can be observed in Fig. 2, which shows erosion results for $M=\{4,8,16\}$ and $r_{w}=\{4,8,16\}$ on a test image. Comparing the upper result row $(M=4)$ to the lower $(M=16)$, it is clear that the sizes of the structuring elements have increased substantially. Meanwhile, going from left $\left(r_{w}=4\right)$ to right $\left(r_{w}=16\right)$ changes adaptivity towards smaller features for adaptivity towards larger features in the data. Thus, the influences of the parameters are as expected: $M$ provides a neighborhood size quantity, and $r_{w}$ needs to be balanced to capture the size of features of interest in the data.

\subsection{Feature Enhancement}

Figure 3 shows the erosion $\left(\varepsilon_{E}(f)\right)$, dilation $\left(\delta_{E}(f)\right)$, opening $\left(\gamma_{E}(f)\right)$, and closing $\left(\psi_{E}(f)\right)$ of the Lena test image, using $M=r_{w}=8$. The opening and closing succeed in enhancing and joining bright and dark features, respectively, directionally without over-stretching over clear borders in the data. This is particularly clear for the feathers and along the brim of the hat.

The presented adaptive elliptical structuring elements range from disks of radius $M / 2$ where no clear local orientation exists in the data to lines of length $2 M+1$ where there is a strong directional structure. For comparison, the opening and closing based on these two extremes are shown in Fig. 4.

For an even more detailed view of the differences, Fig. 5 shows a close-up view of the morphological closing of the upper left part of the hat, depicted contrast-stretched in jet colormap in order to enhance differences. The original image subsection (Fig. 5a) has directional features in the hat, but also contains a noisy region without any directional features in the upper left. We see that the the disk structuring element, which

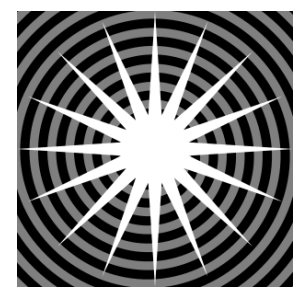

(a)

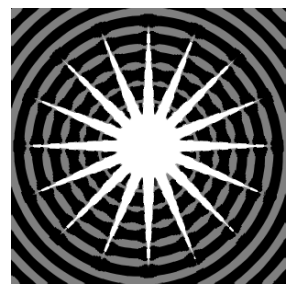

(b)

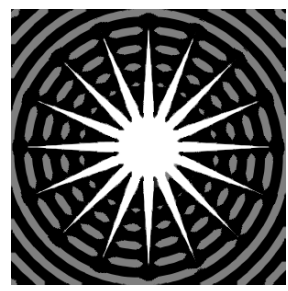

(e)

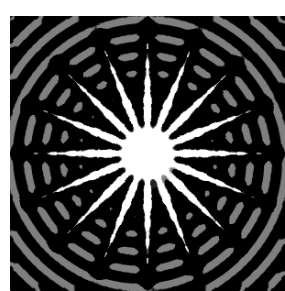

(h)

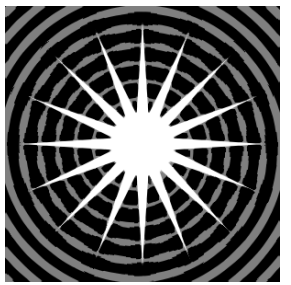

(c)

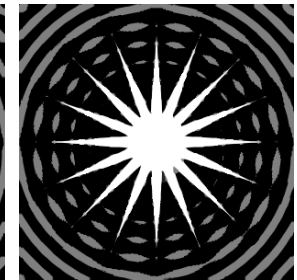

(f)

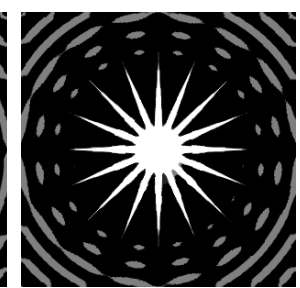

(i)

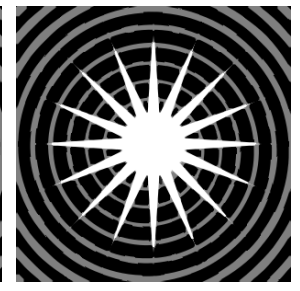

(d)

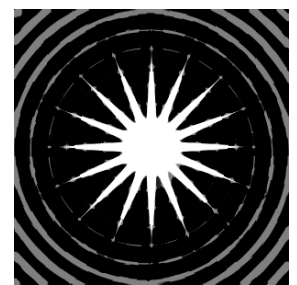

(g)

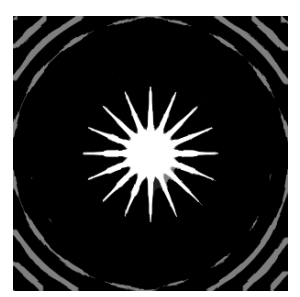

(j)
Figure 2: The star test image (a) and its erosions (b-j) using $M=4,8$, and 16 (top to bottom) and $r_{w}=4,8$, and 16 (left to right).

does not adapt at all to the image structure, gives a crude result (Fig. 5b).

Adaptive line structuring elements (using the per-pixel angles obtained by Eq. (5)) adapt well to existing line features in the data, but also introduce lines in originally smooth regions containing noise (Fig. 5c). We see that the sensitivity of the line structuring elements towards noise in this case comes from the actual shape of the structuring elements rather than their directions, which indicates that this problem needs to be dealt with by using broader structuring elements in such regions.

The presented adaptive elliptical structuring elements (Fig. 5d) provide the trade-off between the two extremes. By utilizing a broader range of shapes for the structuring elements, the operation acts along locally dominant directions in the data without introducing artificial structures in noisy regions where no clear directional structure exists in the original image. This comes at the expense of very thin structures, which are more likely to be erased by the closing using adaptive elliptical structuring elements (as opposed to using adaptive lines). 


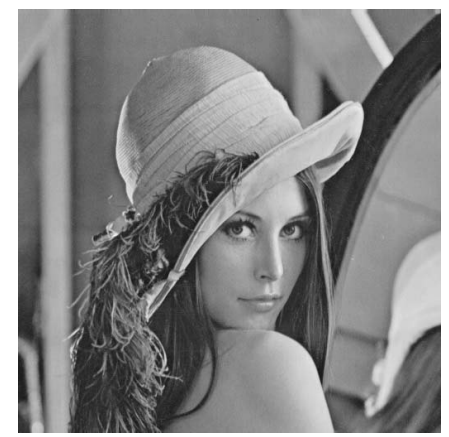

(a)

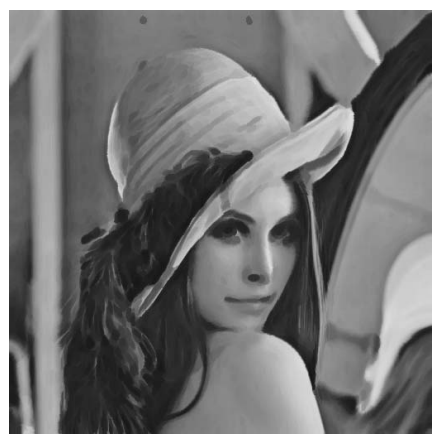

(b)

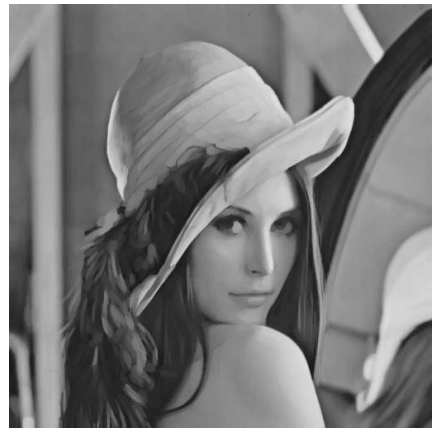

(d)

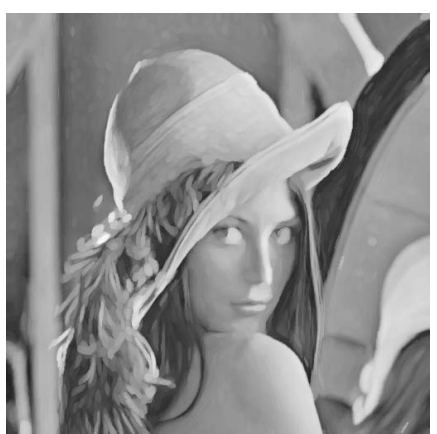

(c)

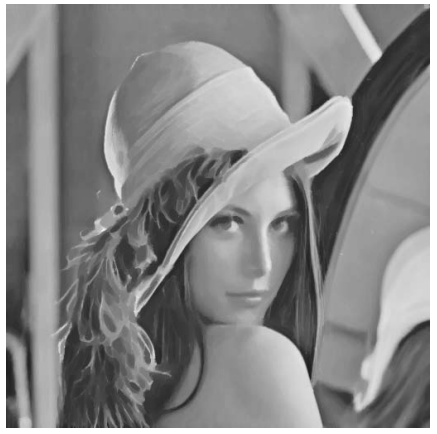

(e)
Figure 3: Operations performed on the Lena image ( $512 \times 512$ pixels) (a) using $M=r_{w}=8$; erosion (b), dilation (c), opening (d), and closing (e).

\subsection{Impact of Noise}

To study the impact of noise, Gaussian white noise with standard deviation $s$ was added to the Lena image $f$, resulting in a noisy image $f_{s}$. The pixel values in the elliptical closing will, as in Fig. 5, generally be higher than in the line closing, since high function values due to the added noise are allowed to affect a larger neighborhood.

For the gradient the situation is different: the fact that adjacent lines in the data may cover completely different sets of pixels causes a highly varying result for noisy data, introducing a substantial amount of artificial edges similar to what we observe already in Fig. 5c. By studying the change in the gradient magnitude of the closed images $\psi\left(f_{s}\right)$ as the amount of noise is increased, the effect of noise on edge information in the image can be more systematically investigated. Figure 6 shows slightly contrast-stretched close-ups of parts of closings $\psi\left(f_{s}\right)$ of the Lena image at noise level $s=0.05$ (Figs. 6a and 6b)

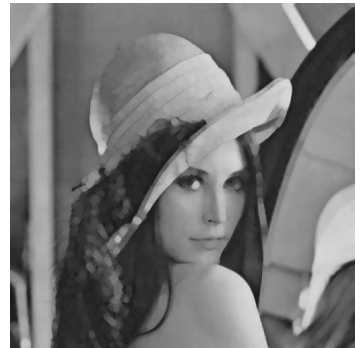

(a)

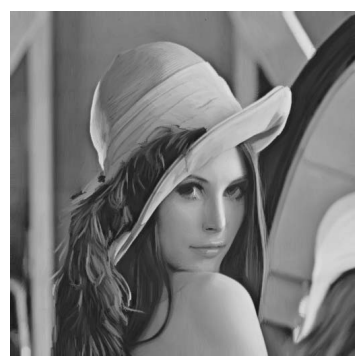

(c)

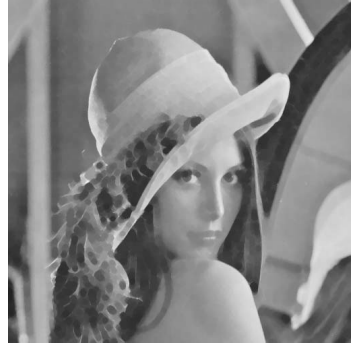

(b)

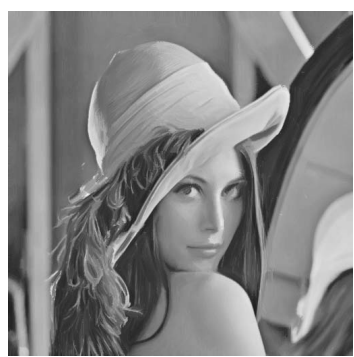

(d)
Figure 4: Openings and closings of the Lena image, using a regular (nonadaptive) disk structuring element of radius $4(a, b)$ and an adaptive line structuring element of length 17 (c, d), corresponding to the two extremes for the adaptive elliptical structuring elements with $M=r_{w}=8$.

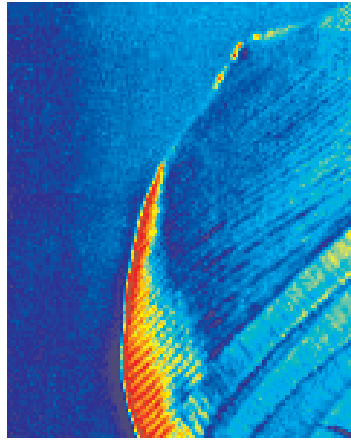

(a)

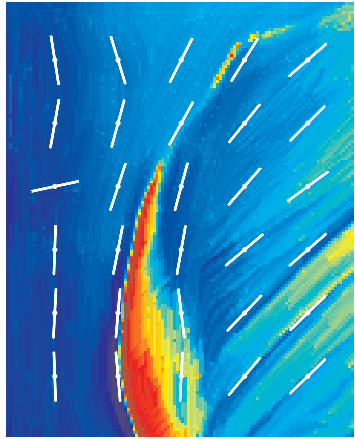

(c)

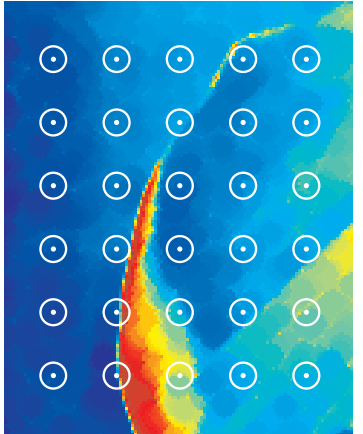

(b)

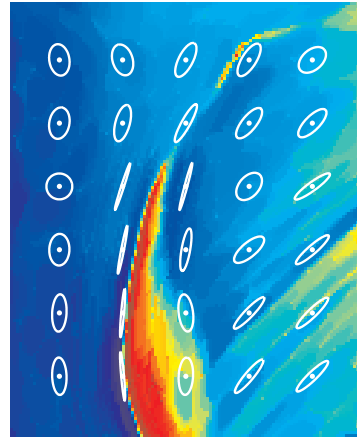

(d)
Figure 5: The upper left part of the hat in the Lena image (a) and its closings using a disk structuring element (b), an adaptive line structuring element (c), and adaptive elliptical structuring elements (d). A subset of the structuring elements used are shown in white. 


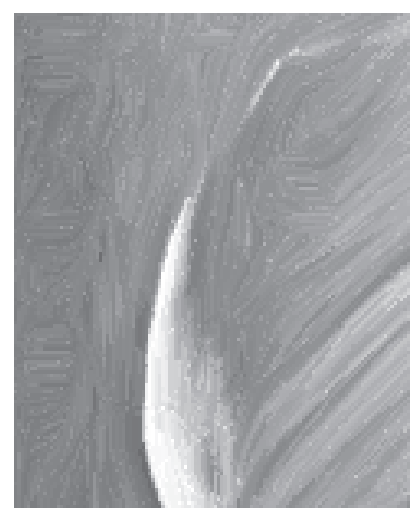

(a)

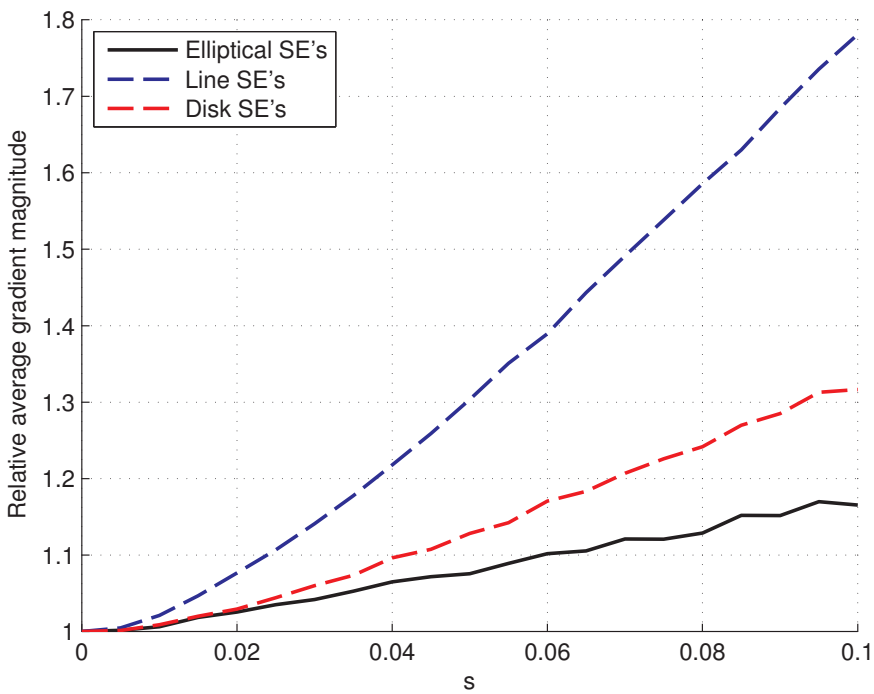

(c)

Figure 6: Results of morphological closings of noise-corrupted data ( $s=0.05$ ) for line structuring elements (a) and elliptical structuring elements (b), and the increasing effect of noise on the gradient magnitude in filtered images (c).

and a plot of the ratio between the average gradient magnitudes for the closed noisy images and the closed original image, i.e. the ratio $\left(\psi\left(f_{s}\right) / \psi(f)\right)$, as the standard deviation $s$ of the added noise increases from 0 to 0.1 (Fig. 6c). Some elliptical structuring elements can be identified in Fig. 6b, but the lines in Fig. 6a are much more distinct. This visual observation is more systematically validated in Fig. $6 \mathrm{c}$, which indeed shows that the noise causes a substantially larger increase of edge structure in the line-filtered image than in the ellipse-filtered image.

\subsection{Practical Application}

Our final example is of a more practical nature. In Fig. 7a we see 3D profile (range) data of a surface crack in casted steel. The crack has clear directional characteristics, but is quite noisy. By enhancing the crack signature we can more easily distinguish it from the rest of the surface, which simplifies automated crack detection and allows for a more accurate estimation of crack length. After performing an opening using elliptical structuring elements (Fig. 7b) on the image in question the

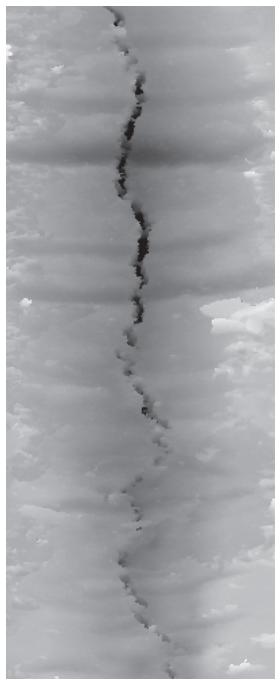

(a)

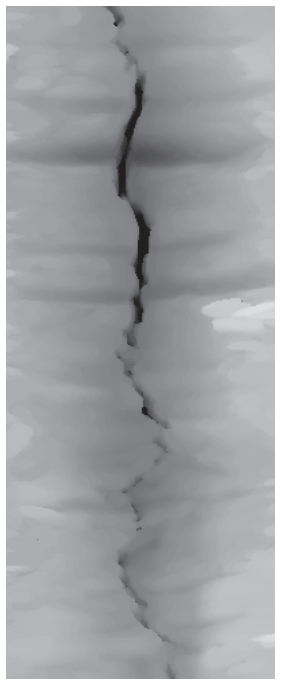

(b)

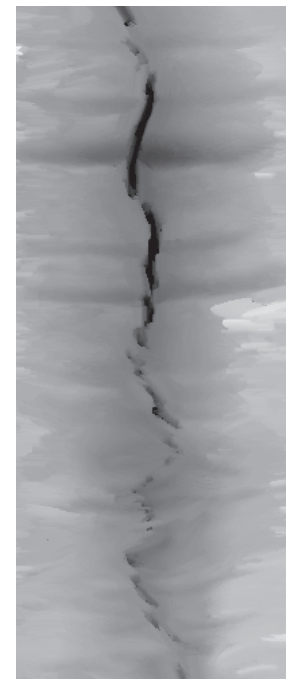

(c)
Figure 7: A noisy vertical surface crack (a) and its opening using adaptive ellipses (b) and adaptive lines (c) using $M=r_{w}=10$. The elliptical structuring elements manage to enhance the crack as good as the line structuring elements. Also, note that horizontal trenches, known as oscillation marks, are not noticeably affected.

crack signature is indeed clearly enhanced. In particular, the operation efficiently joins crack sections that were originally split apart by noise, without noticeably affecting the horizontal trenches (known as oscillation marks).

This example demonstrates how the presented methods can be used in challenging applications in computer vision. Enhancement of the clearly directional crack is as good for the elliptical structuring elements as for the more extreme line structuring elements (Fig. 7c), but the advantage of the presented method is that we do not risk introducing artificial lines as a result of noise in the data, as was discussed in Section 4.3. In particular, the type of distinct dark lines that appear in the originally flat but noisy upper left region of Fig. $6 \mathrm{a}$ as a result of the shapes of the line structuring elements would in this application likely be falsely interpreted as cracks. Elliptical structuring elements therefore make the system less sensitive to noise, which is highly desirable for an automated industrial system subject to environmental factors such as vibrations, dust, reflections, etc.

\section{Discussion}

We have shown how the concepts of tensors and morphology can be combined into adaptive morphology in a straightforward manner. Previous work has touched the subject but not fully utilized the information held by the Local Structure Tensor (LST). By considering the relation between the eigenvalues as well as the direction obtained from the LST, edge dominancy information is automatically included when assigning a structuring element to a pixel.

The results show that the structuring elements indeed become line-shaped (or close to line-shaped) where there are strong dominant directions in the data, i.e. near edges. In addition, 
when processing regions further away from distinct edges the method adapts dynamically to a non-directional approach by transforming the structuring element into smaller disks, which avoids a distinct geometrical bias from the filter itself in such locations. In particular, this is true when data contains random noise, in which case the noise level affects the morphological operations (which are based on minimum and maximum values within neighborhoods) but does not cause artificial edges in the data resulting from biases from the structuring element.

If the user needs to emphasize extremely thin features but does not care that similar features risk being added from noise (e.g. if the Signal to Noise Ratio is known to be very high) line structuring elements may very well be the best choice. For cases where noise is more of an issue, however, and in particular where thin artificial structures are unwanted, the presented adaptive elliptical structuring elements should be preferable.

The method relies on no more than two input parameters: the maximum semi-major axis $M$ and the radial bandwidth for structure estimation $r_{w}$. The parameters are quite intuitive and are related to real measures in the image; $M$ sets the sizes of the structuring elements while $r_{w}$ is related to the size of orientational features of interest. These two can in many cases even be set to the same number, since the possible reach of the assigned structuring elements then corresponds to the region considered for structure estimation. If needed, the level of Gaussian prefiltering when calculating the gradient can of course also be set by changing the underlying parameter $\sigma^{\prime}$ to match the level of noise expected by the user, but for most cases a small smoothing $\left(\sigma^{\prime}<1\right)$ should suffice. Hence, the presented morphological processing is reasonably easy to use even without deep insight into the underlying framework.

Regarding efficiency, the LST calculations are based on standard convolutions - a standard process which is usually implemented highly efficiently. The eigenvalues of the LST can in lower dimensions be calculated from closed-form expressions, and can therefore be handled quite efficiently as well (but will of course need more computational power in higher dimensions). As for the morphological operations, a Look-Up Table (LUT) allows for a reasonably efficient implementation. In systems where the adaptive morphological operations should be repeated or otherwise performed several times, the LUT can be calculated once (off-line) and then loaded into on-line applications, reducing the computational load for on-line systems at the cost of memory.

\section{Conclusion}

The adaptive elliptical structuring elements are robust to noise and are automatically adjusted to the local edge information (or lack thereof) in the data. They constitute a dynamic bridge between the two extremes; disk and (direction-adaptive) line structuring elements.

We have demonstrated how the adaptive elliptical structuring elements can be applied to both gray-scale images and 3D profile data used in industrial applications, allowing for enhancement of directional structures within the data.

\section{Future work}

In this work, we set the structuring elements by setting two parameters: the maximum semi-major axis $M$ and the filter bandwidth $r_{w}$ used to obtain the local structure tensor. In the future we intend to investigate the possibility to set those parameters adaptively as well. This could be done by calculating the LST at different scales (for different values of $r_{w}$ ) and select a value for $M$ that captures the scale at which the LST captures enough information. Alternatively, other approaches for estimating image structure, where there is an even stronger relation to the variation in the different directions (e.g. where crossings can be separated from points), could be used to scale $M$ suitably.

Future work should also consider estimating the level of noise in the input image in order to define a proper level of pre-smoothing for calculating the gradient. This would allow for a more proper handling of thin structures in noise-free data.

Finally, there is no reason why the presented results cannot be generalized for higher dimensions, enabling processing of voxel information.

\section{References}

Beucher, S., 2007. Numerical residues. Image and Vision Computing 25, 405415.

Bouaynaya, N., Charif-Chefchaouni, M., Schonfeld, D., 2008. Theoretical foundations of spatially-variant mathematical morphology part i: Binary images. IEEE Transactions on Pattern Analysis and Machine Intelligence 30, 823-836.

Bouaynaya, N., Schonfeld, D., 2006. Spatially variant morphological image processing: theory and applications, in: Proceedings of SPIE, pp. 673-684.

Bouaynaya, N., Schonfeld, D., 2008. Theoretical foundations of spatiallyvariant mathematical morphology part ii: Gray-level images. IEEE Transactions on Pattern Analysis and Machine Intelligence 30, 837-850.

Breuß, M., Burgeth, B., Weickert, J., 2007. Anisotropic continuous-scale morphology, in: Martí, J., Benedí, J., Mendonça, A., J., S. (Eds.), Pattern Recognition and Image Analysis. Part II. Lecture Notes in Computer Science, Springer, Berlin. pp. 515-522.

Cammoun, L., Castaño-Moraga, C., Muñoz-Moreno, E., Sosa-Cabrera, D., Acar, B., Rodriguez-Florido, M., Brun, A., Knutsson, H., Thiran, J., 2009. A review of tensors and tensor signal processing, in: Tensors in Image Processing and Computer Vision, Springer. pp. 1-32.

Charif-Chefchaouni, M., Schonfeld, D., 1994. Spatially-variant mathematical morphology, in: IEEE International Conference on Image Processing (ICIP), IEEE. pp. 555-559.

Cuisenaire, O., 2006. Locally adaptable mathematical morphology using distance transformations. Pattern recognition 39, 405-416.

Curic, V., Luengo Hendriks, C., Borgefors, G., 2012. Salience adaptive structuring elements. IEEE Journal of Selected Topics in Signal Processing 6, 809-819.

Debayle, J., Pinoli, J., 2005. Spatially adaptive morphological image filtering using intrinsic structuring elements. Image Analysis \& Stereology 24, 145 158 .

Dokládal, P., Dokládalová, E., 2008. Grey-scale morphology with spatiallyvariant rectangles in linear time, in: Advanced Concepts for Intelligent Vision Systems, Springer. pp. 674-685.

Lerallut, R., Decencière, É., Meyer, F., 2007. Image filtering using morphological amoebas. Image and Vision Computing 25, 395-404.

Maragos, P., Vachier, C., 2009. Overview of adaptive morphology: trends and perspectives, in: 16th IEEE International Conference on Image Processing (ICIP), IEEE. pp. 2241-2244.

Matheron, G., 1975. Random sets and integral geometry. volume 1. Wiley, New York.

OpenCV, v.2.3, 2011. Open source computer vision library. http://opencv . org. 
Rieger, B., Van Vliet, L., 2004. A systematic approach to $n$ d orientation representation. Image and Vision Computing 22, 453-459.

Roerdink, J., 2000. Group morphology. Pattern Recognition 33, 877-895.

Roerdink, J., 2009. Adaptivity and group invariance in mathematical morphology, in: 16th IEEE International Conference on Image Processing (ICIP), IEEE. pp. 2253-2256.

Roerdink, J., Heijmans, H., 1988. Mathematical morphology for structures without translation symmetry. Signal Processing 15, 271-277.

Serra, J., 1982. Image analysis and mathematical morphology. Academic Press, London.

Serra, J., 1988. Image analysis and mathematical morphology. Vol. 2. Academic Press, New York, NY

Serra, J., 2005. Viscous lattices. Journal of Mathematical Imaging and Vision 22, 269-282.

Shih, F., Cheng, S., 2004. Adaptive mathematical morphology for edge linking. Information sciences 167, 9-21.

Shih, F., Gaddipati, V., 2003. General sweep mathematical morphology. Pattern recognition 36, 1489-1500.

Tankyevych, O., Talbot, H., Dokládal, P., Passat, N., 2009. Direction-adaptive grey-level morphology. application to $3 \mathrm{~d}$ vascular brain imaging, in: 16th IEEE International Conference on Image Processing (ICIP), IEEE. pp. 2261-2264.

Verdú-Monedero, R., Angulo, J., 2008. Spatially-variant directional mathematical morphology operators based on a diffused average squared gradient field, in: Advanced Concepts for Intelligent Vision Systems, Springer. pp. 542-553.

Verdú-Monedero, R., Angulo, J., Serra, J., 2009. Spatially-variant anisotropic morphological filters driven by gradient fields. Mathematical Morphology and Its Application to Signal and Image Processing , 115-125.

Verdú-Monedero, R., Angulo, J., Serra, J., 2011. Anisotropic morphological filters with spatially-variant structuring elements based on image-dependent gradient fields. Image Processing, IEEE Transactions on 20, 200-212. 